\title{
Desempenho Motor e Quedas: um Estudo Comparativo entre Idosos Cadastrados no Programa Saúde da Família, no Município de Vitória de Santo Antão-PE
}

\author{
Motor Performance and Falls: a comparative study among elderly \\ registered in the family health program, in Vitória de Santo Antão-PE
}

\author{
Lisandra Delfino Soares ${ }^{1}$ \\ Florisbela Arruda Campos ${ }^{2}$ \\ Maria Das Graças Rodrigues Araújo ${ }^{3}$ \\ Ana Patrícia Siqueira Falcão ${ }^{4}$ \\ Bruna Rafaela Dornelas Lima ${ }^{5}$ \\ Danielle Ferreira Siqueira ${ }^{5}$ \\ Silvana Gonçalves Arruda ${ }^{6}$ \\ Zelyta Pinheiro de Faro ${ }^{6}$
}

\section{RESUMO}

Objetivo: Analisar a relação entre o risco de quedas e o desempenho das habilidades motoras de idosos, cadastrados no Programa Saúde da Família - PSF, zona urbana, do Município de Vitória de Santo Antão, Região da Zona da Mata do Estado de Pernambuco. Material e Métodos: Estudo do tipo epidemiológico, transversal de campo do tipo descritivo-analítico, observacional e de inquéritos. Participaram desta pesquisa 235 idosos com idade superior ou igual a 60 anos, do gênero masculino e feminino, residentes em Vitória-PE. O medo de cair foi avaliado por meio da escala de eficácia de quedas FES-I (Falls efficacyscale-International). Para a análise do desempenho motor foram utilizados quatro testes motores: Time Up\&Go, Índice de Tinetti, Alcance Funcional e o Apoio Unipodal, avaliados por tempo e equilibrio. As associações foram verificadas segundo a correlação de Spearman. Resultados: Os dados do presente estudo apontam para uma ocorrência maior de quedas entre o gênero feminino do que no masculino. Os idosos que relataram quedas recorrentes no último ano apresentaram maior comprometimento funcional, independentemente, do sexo. A associação entre a pontuação na FES-I e o número de quedas no ano anterior foi estatisticamente significativa $(r=0,602 ; p=0,005)$. Conclusão: Os resultados da presente pesquisa apontaram uma associação entre quedas e a capacidade funcional independente do sexo.

DESCRITORES: Avaliação Geriátrica. Acidentes Por Quedas. Atividade Física.

\begin{abstract}
Objective: To analyze the relation between the risk of falls and elderly's motor skills performance (registered in programa de saúde da famiia - family health program - in the urban area of Vitória de Santo Antão, in Zona da Mata, in Pernambuco state). Material and Methods: Epidemiological, transversal, descriptive and analytical field study, being observational and survey-like. 235 elderly residents, both male and female (60 year old or so), residents in VitóriaPE participated in this research. Fear of falling and falls occurrence were evaluated by FES-I (falls efficacy scale-International). Four motor tests were used to analyze motor performance: Time Up\&Go Tinetti's Index, Functional Reach and Unipodal Support, evaluated for time and balance. The associations were verified according to Spearman's correlation. Results: The data of this current study indicate a higher occurrence of falls among women. Elders who reported recurrent falls in the previous year presented a bigger functional difficulty, regardless of their genre, compared to the ones that reported only one fall in the previous year and the ones that reported none. The association between FES-I score and the number of falls in the previous year was statistically significant $(r=0,602$ $p=0,005)$. Conclusion: The results of the current survey showed an association between falls and functional ability, regardless the genre.
\end{abstract}

DESCRIPTORS: Geriatric Assessment. Accidental Falls. Physical Activity.

1- Mestranda em Saúde Humana e Meio Ambiente da Universidade Federal de Pernambuco-UFPE/CAV.Vitória de Santo Antão-PE

2- Docente titular Doutora do Núcleo de Educação Física e Ciência do Esporte do Centro Acadêmico da Vitória da Universidade Federal de Pernambuco-UFPE Vitória de Santo Antão-PE

3- Docente Doutor do Departamento de Fisioterapia do Centro de Ciências da Saúde da Universidade Federal de Pernambuco-UFPE, Vitória de Santo Antão-PE

4- Docente Doutor do Instituto Federal de Educação, Ciência e Tecnologia, Campus Vitória - PE e da Escola Superior de Educação Física - ESEF/UPE, Vitória de Santo Antão-PE

5- Mestranda em Saúde Humana e Meio Ambiente da Universidade Federal de Pernambuco-UFPE/CAV.Vitória de Santo Antão-PE

6- Docente Adjunto Doutor do Núcleo de Nutrição do Centro Acadêmico da Vitória da Universidade Federal de Pernambuco-UFPE, Vitória de Santo Antão-PE 
$\mathrm{O}$ crescimento da população de idosos, em números absolutos e relativos, é um fenômeno mundial e está ocorrendo em nível sem precedentes ${ }^{1}$. Os idosos constituem o segmento da população que mais cresce na atualidade. Entre 1991 e 2000, o número de habitantes com 60 a 69, 70 a 79 e 80 ou mais anos aumentou cerca de duas a quatro vezes mais $(28 \% \%, 42 \%$ e $62 \%$, respectivamente) que a população mais jovem do país $-14 \%{ }^{2}$.

Apesar de ser um processo natural, o envelhecimento submete o organismo a diversas alterações anatômicas e funcionais, com repercussões nas condições de saúde do idoso ${ }^{3}$.

As causas mais comuns para a perda da capacidade funcional no idoso são a imobilidade e a inatividade (sedentarismo) ${ }^{4}$. Idosos independentes para as atividades de vida diária (AVD), autônomos e satisfeitos com as relações familiares e com os amigos, apresentavam fator preditivo independente para o envelhecimento saudável para ambos os gêneros ${ }^{4}$.

A independência do idoso para realizar as AVD's é prejudicada com o avançar da idade, reduzindo, desta forma, a qualidade de vida e aumentando o risco de dependência. A diminuição da capacidade funcional é referida como fator de aumento no risco de quedas, principalmente devido ao comprometimento na realização de tarefas do dia a dia, com limitações de força muscular, equilíbrio, marcha e mobilidade ${ }^{5}$. No Brasil, $30 \%$ dos idosos caem pelo menos uma vez ao ano, sendo as quedas causadas por uma rede de fatores: herança genética, história da atividade, fatores socioeconômicos, personalidade, educação, autoconfiança, doenças não diagnosticadas ${ }^{6}$, uso de medicamentos e/ou fatores relacionados ao ambiente como, áreas pouco iluminadas ${ }^{7}$.

A incidência de quedas em idosos trazem consequências de gravidades variáveis, estando relacionadas tanto aos aspectos físico e psicológico do indivíduo, como também nos econômicos e sociais $^{8}$. Em virtude do envelhecimento encontrar-se em estágios avançados, quanto maior a idade do indivíduo, maior será o risco de quedas. Em um estudo realizado em comunidades americanas, com idosos acima de 65 anos, foi visto que 30\% desses sofreram quedas pelo menos $1 \mathrm{vez}$ ao ano, sendo que $40 \%$ desses idosos apresentavam idade superior a 80 anos.

As quedas, normalmente, encontram-se relacionadas ao desequilíbrio durante a marcha, sendo bastante necessário uma avaliação desta associação, para analisar o prognóstico e, quase sempre, estão associadas ao desequilíbrio durante a marcha. Portanto, uma avaliação deste fenômeno é decisiva para o prognóstico e para investigação de tratamentos futuros ${ }^{9}$.

A realização de estudos desta natureza são importantes, pois possibilitam a aquisição de dados sobre o perfil de idosos mais predispostos a queda, possibilitando uma abordagem preventiva relacionada as necessidades individuais dos idosos, promovendo a esses uma melhor qualidade de vida, além de diminuir os custos com a saúde pública do país ${ }^{8,11,12}$. A prevenção de quedas pode reduzir o número de idosos dependentes .

Apesar da alta prevalência de quedas entre os idosos, no Brasil poucos são os estudos epidemiológicos sobre esse assunto. Desta forma, o presente estudo visa investigar a incidência de quedas referida pelos idosos e relacioná-las com a capacidade funcional destes.

\section{MATERIAL E MÉTODOS}

Trata-se de um estudo do tipo epidemiológico, transversal de campo do tipo descritivo-analítico, observacional e de inquéritos sobre quedas e avaliação de seus fatores de risco numa população de idosos.

Este estudo foi realizado na Cidade de Vitória de Santo Antão, localizada na Zona da Mata Sul do Estado de Pernambuco, distante $55 \mathrm{~km}$ da Cidade do Recife. A coleta foi realizada no período de março à setembro de 2010, nas 20 unidades do Programa Saúde da Família (PSF) da zona urbana da cidade (Santana, Caiçara, Conceição, Cidade de Deus, Lagoa Redonda, Doutor Alvinho, Lídia Queiroz, Maranhão, Redenção, Amparo, Jardim Ipiranga, Maués, Alto José Leal, Programa de Agentes Comunitários de Saúde (PACS) PACS Urbano I, PACS Urbano II, Matadouro, Mário Bezerra, Cajueiro, Bela Vista I e Bela Vista II) conforme informação do Sistema de Informação Básica (SIAB).

Participaram desta pesquisa 235 idosos com idade superior ou igual a 60 anos, do gênero masculino e feminino, residentes em Vitória de Santo Antão -PE e cadastrados nas Unidades de Saúde da Família (USF), referentes à área de abrangência de sua residência, da zona urbana da cidade. Foram excluídos do projeto idosos com: demência senil, doença ou sequela neurológica que interferisse na capacidade de equilíbrio corporal; doenças ortopédicas que afetassem nas AVDs ou na locomoção; cegueira ou déficit visual grave que comprometesse a locomoção independente; 
portadores de órteses ou dependentes de cadeiras de rodas e hipertensão arterial sistêmica moderada ou grave à hora do exame ${ }^{14}$.

Os idosos foram submetidos a uma avaliação do desempenho motor (DM) e a um questionário sobre quedas, realizados por fisioterapeutas e estudantes do curso de graduação em nutrição, previamente capacitados.

A análise do desempenho motor consistiu de quatro testes (Time up\&go-TUG, Índice de Tinetti-IT, Alcance Funcional-AF e Apoio Unipodal-AU) que avaliavam entre outros o equilíbrio, a mobilidade e a flexibilidade. Durante o TUG foi mensurado o tempo, em segundos, o tempo que o idoso levava para levantar da cadeira, andar uma distância de $3 \mathrm{~m}$, dar a volta, caminhar de volta e sentar na cadeira ${ }^{15}$; representando, desta forma um conjunto de atividades rotineiras importantes para a independência funcional. Os idosos realizavam o teste uma vez, para compreendê-lo e, somente na segunda tentativa, o tempo era cronometrado.

A utilização do IT, consistiu de duas escalas: de equilíbrio e de marcha. A primeira possui 09 itens: equilíbrio sentado, levantando, tentativas de levantar, assim que levanta, equilíbrio em pé, teste dos três tempos, olhos fechados, girando $360^{\circ} \mathrm{e}$ sentando. A segunda possui 07: início da marcha, comprimento e altura dos passos, simetria dos passos, continuidade dos passos, direção, tronco e distância dos tornozelos. A pontuação total do índice é de 28 pontos. Pontuação menor que 19 indica risco cinco vezes maior de quedas ${ }^{16,17}$.

O teste AF ("Functional Reach Test") ou "teste do alongamento funcional" foi desenvolvido por DUNCAN et al (1990) ${ }^{9}$. A avaliação foi realizada usando uma fita métrica, fixada na parede, na altura do ombro do paciente, o qual fica de pé, na posição de lado, em relação à parede, com os pés separados e alinhados em relação aos ombros e com os braços elevados a 90 graus de flexão, com extensão de punho e dedos. O paciente foi instruído a alongar o corpo para frente o máximo possível, sem movimentar os pés, sem perder o equilíbrio, sem dar um passo e sem tocar na parede ou na fita. Foram realizadas três medições, e o resultado final foi registrado pela média das aferições. Deslocamentos menores que $15 \mathrm{~cm}$ indicam risco de quedas.
$\mathrm{O}$ teste $\mathrm{AU}$ avalia o tempo que os idosos conseguem permanecer em apoio unipodal, com os olhos abertos e com os olhos fechados, avaliando deste modo seu equilíbrio; tempos inferiores a 5 segundos sugerem maior probabilidade de quedas $^{19}$.

Os idosos foram questionados sobre medo de cair e a ocorrência de quedas. A avaliação do medo de cair foi realizada pela escala de eficácia de quedas FES-I (Falls efficacyscale-International $)^{20}$ com escore máximo de 64 pontos. Os escores inferiores a 23 pontos indicam um maior medo de cair. Com relação a ocorrência de quedas, foi realizado um questionário, em que se perguntava sobre o número de quedas no último ano e os motivos associados.

Este trabalho foi realizado respeitando os aspectos éticos, aplicando-se o Termo de Consentimento Livre e Esclarecido. O projeto de pesquisa foi submetido e aprovado pelo Comitê de Ética em Pesquisa envolvendo seres humanos do Centro de Ciências da Saúde da Universidade Federal de Pernambuco (CEP/CCS/UFPE): Protocolo $n^{0}: 241 / 09$.

Quanto ao método estatístico, foi realizado inicialmente uma análise descritiva por meio de mediana, valores mínimos e máximos e uma distribuição de frequência. Posteriormente, foi realizada uma correlação de Spearman entre os indicadores nutricionais e os testes de desempenho motores. Todas as análises foram realizadas no Programa SPSS versão 18.0. O nível de significância adotado foi de $5 \%$.

\section{RESULTADOS}

Dos 235 idosos que participaram do estudo, $77,9 \%$ pertenciam ao sexo feminino $(n=183)$ e $22,1 \%$ ao sexo masculino $(n=52)$. A idade dos idosos variou de 60 a 94 anos, com uma idade média de $69,09 \pm 7,13$ anos. Para o sexo feminino ( $n=183$ ), a média etária foi de 67,84 $\pm 6,07$ anos (60 a 84 anos). Para o sexo masculino $(n=52)$ foi de 73,5 \pm 8,74 anos (60 a 94 anos).

Foi observado um alto índice de queda entre as idosas participantes da pesquisa, como pode ser visto na figura 1. 
Figura 1: Frequência de quedas entre idosos do sexo feminino, no ano de 2010, nas 20 unidades do Programa Saúde da Família (PSF) da zona urbana da cidade de Vitória de Santo Antão - PE.

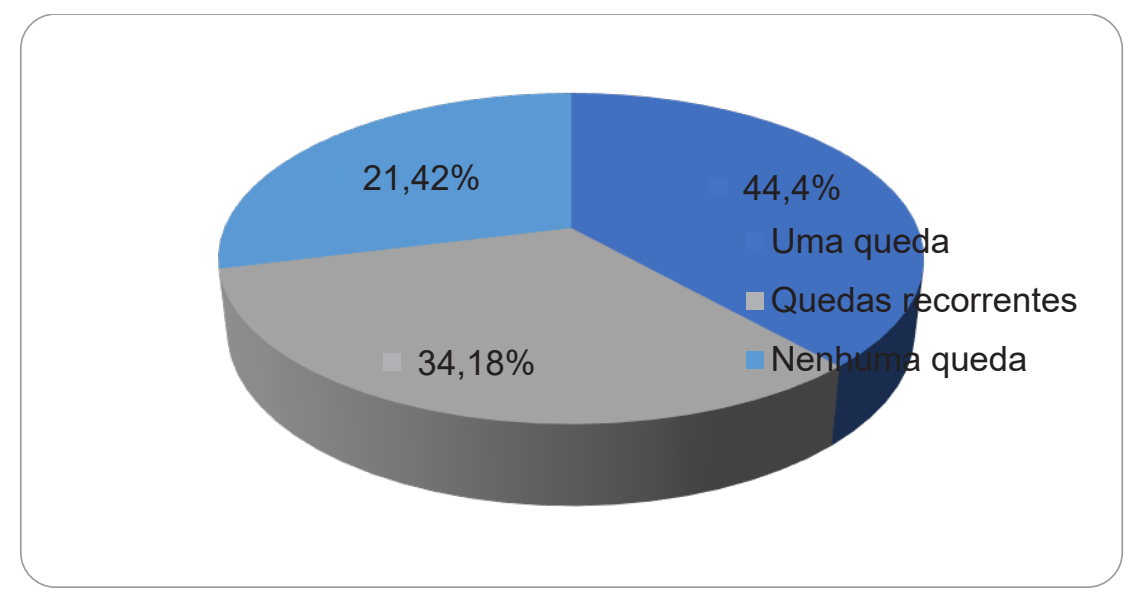

Dos idosos do sexo masculino $(n=52)$, $57,7 \%(n=30)$ caíram no último ano, sendo que $38,4 \%(n=20)$ sofreram uma queda, $32,7 \%(n=17)$ sofreram quedas recorrentes e $28,9 \%(n=15)$ não sofreram quedas durante o último ano (Figura 2).

Na Figura 3 é possível observar que idosos que relataram quedas recorrentes no último ano, apresentaram maior comprometimento funcional, independentes do sexo, quando comparados aos que relataram terem tidos apenas uma queda no último ano e aos que não tiveram nenhuma queda.

Pela análise da correlação de Spearman foi possível encontrar uma associação significativa e moderada entre a pontuação na FES-I e o número de quedas no ano anterior $(r=0,602$; $p=0,005)$.

Figura 2: Frequência de quedas entre idosos do sexo masculino no ano de 2010, nas 20 unidades do Programa Saúde da Família (PSF) da zona urbana da cidade de Vitória de Santo Antão - PE. 


\section{Figura 3: Distribuição entre números de quedas e comprometimento funcional (TUG) de idosos cadastrados no PSF - Vitória - PE.}

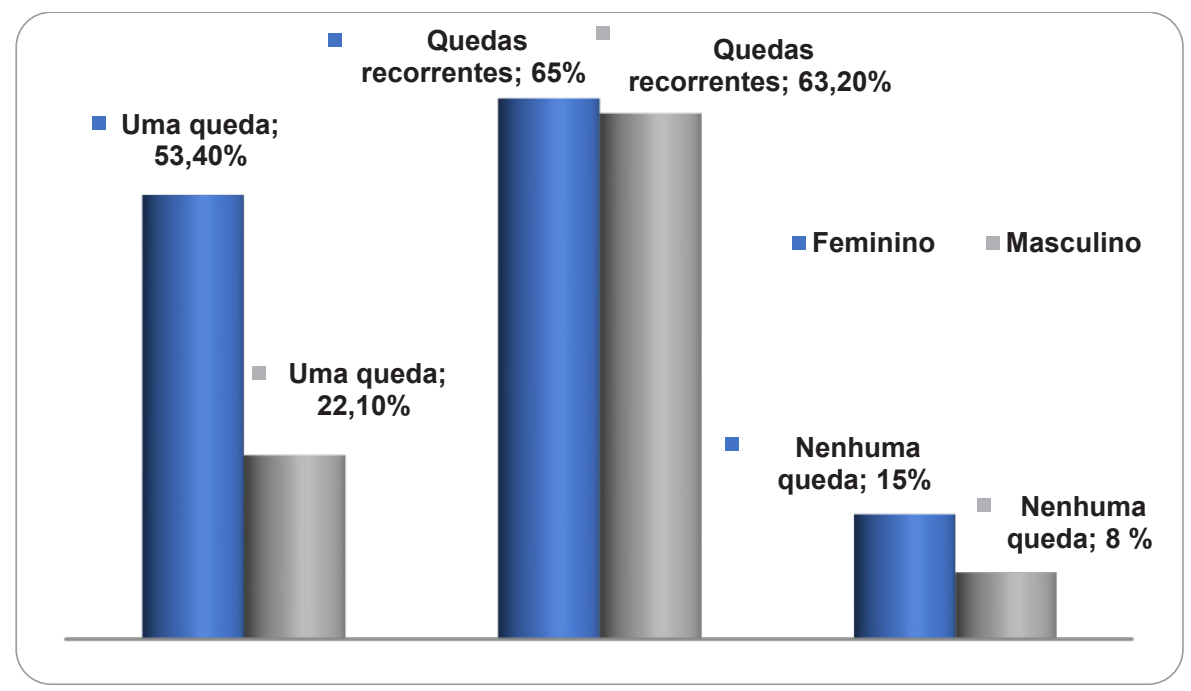

\section{DISCUSSÃo}

Estudos realizados tanto em países em desenvolvimento, quanto em países desenvolvidos indicam um alto índice de quedas no grupo de idosos $21,22,23,24$

Os dados do presente estudo apontam para uma ocorrência de quedas maior entre o gênero feminino do que no masculino ${ }^{23}$. Nesse artigo são citados como indicativos responsáveis por esta diferença o sedentarismo, o uso de psicotrópicos e o isolamento.

As quedas em idosos podem ter causas variadas, na literatura encontra-se os fatores responsáveis classificados como intrínsecos, os decorrentes de alterações fisiológicas comuns ao envelhecimento ou doenças, usos de medicamentos; e extrínsecos, como aqueles fatores relacionados ao meio ambiente e social em que 0 idoso vive ${ }^{24}$. A análise da identificação de causas de quedas entre idosos torna-se bastante complexa, já que os fatores intrínsecos e extrínsecos podem encontra-se associados. Normalmente, os problemas com o ambiente trazem mais sequelas quanto maior for o grau de vulnerabilidade do idoso, ou seja, quanto mais comprometimento físico ou mental tiver o idoso maior a chance de ocorrer as quedas.

Em 2001, foi realizado um de um estudo comparativo entre mulheres jovens e idosas e neste estudo foi observado que o envelhecimento tem relação com uma diminuição da força muscular em idosos, mesmo com a prática regular de atividade física. A diminuição da força muscular pertinente aos idosos acarreta uma maior probabilidade de quedas $^{26}$

Outro estudo analisou a relação de déficit de força muscular com o aumento do número de quedas, verificando-se que o equilíbrio do corpo se encontra de acordo com as condições articulares e musculares do indivíduo. Assim como, em situação de quedas iminente, o restabelecimento do equilíbrio irá depender entre outros fatores da recuperação da força da musculatura dos membros inferiores ${ }^{27}$. Em uma pesquisa realizada em 2008, os autores chegaram a conclusão que quanto melhor a força muscular dos membros inferiores dos idosos melhor será sua capacidade de recuperar-se de uma queda. Esses resultados encontram-se de acordo com o presente estudo, no qual foi verificado que $62,9 \%$ dos idosos que apresentaram um comprometimento funcional relataram quedas recorrentes ${ }^{28}$

O medo de cair é outro fator que pode agravar o número de quedas. O medo de cair gera uma insegurança nos familiares dos idosos, agindo de forma protetora e consequentemente, privando os idosos de realizarem suas atividades diárias. Isto pode acarretar num aumento do sedentarismo neste grupo populacional, levando a situações de insegurança e isolamento, trazendo com isso um prejuízo na qualidade de vida dos idosos ${ }^{29,30}$.

No nosso estudo foi encontrada uma correlação positiva entre o medo de cair avaliado pela FES-I e o número de quedas. Situação semelhante foi verificada em estudos anteriores, quando foi visto uma associação positiva entre o medo de cair (FES-1) e a dependência em atividades de vida diária ${ }^{31}$ 
Esta pesquisa aponta a necessidade de garantir a prática de atividades preventivas na redução do índice de quedas. Nesta perspectiva, esse trabalho deverá ser em conjunto com outros profissionais da saúde, por meio de palestras, atividades e práticas de atividades físicas, promovendo deste modo uma melhora do equilíbrio e da marcha dos idosos e, consequentemente, trazendo uma melhoria na qualidade de vida.

\section{REFERÊNCIAS}

1. IBGE. Perfil dos idosos responsáveis pelos domicílios no Brasil 2000. Estudos e Pesquisas Informação Demográfica e Socioeconômica, 2002;n.9. Rio de Janeiro.

2. Lima-Costa MF, Barreto SM, Giatti L. Condições de saúde, capacidade funcional, uso de serviços e gastos com medicamentos da população idosa brasileira: um estudo descritivo baseado na PNAD. Cad Saúde Pública. 2003;19(3):735-43.

3. Campos MTFS, Monteiro, JBR.; Ornelas, PRC. Fatores que afetam o consumo alimentar e a nutrição do idoso. Revista de Nutrição. 2000;13(3):157-165

4. Moraes JLD, Azevedo e Souza, VB. Factors associated with the successful aging of the socially-active elderly in the metropolitan region of Porto Alegre. Revista Brasileira de Psiquiatria.2005; 27(4):302-308.

5. Pereira SRM et al., Quedas em Idosos. Sociedade Brasileira de Geriatria e Gerontologia Projeto Diretrizes; Jun 2001.

6. Kauffman TL. Manual de reabilitação geriátrica. Rio de Janeiro: Guanabara Koogan; 2001.

7. Perracini MR. Prevenção e Manejo de Quedas. In: Ramos LR. Guia de Geriatria e Gerontologia. São Paulo: Manole; 2005; p.193-208.

8. PerraciniNI MR, Ramos LLR. Fatores associados a quedas em um coorte de idosos residentes na comunidade. Rev Saúde Pública. 2002; 36 (6):709-16

9. Mathias S, Nayak USL, Isaacs B. Balance in elderly patients: the "get-up and go" test. Arch. Phys. Med. Rehabil.1986; 67:387-389.

10. Tinetti ME,Speechley M. Prevention of falls among the elderly. $\mathrm{N}$ Engl J Med. 1989; 320(16): 1055-9.

11. Shimada H, Obuchi S,Furana T, Suzuki T. New intervention program for preventing falls among frail elderly people. Am J Phys Med Rehab. 2004; 83(7):493-9.

12. Garcia R, Leme MD, Garcez-Leme LE. Evolution of Brazilian elderly with hip fracture secondary to a fall. Clinics (São Paulo). 2006; 61(6):539-44.

13. Almeida Filho N,Rouquayrol MZ. 2003. Elementos de Metodologia Epidemiológica. In: ROUQUAYROL, M.Z.; ALMEIDA FILHO, N. Epidemiologia e Saúde. 6.ed. Rio de Janeiro: Medsi, cap.6. p.149-177.

14. Tavares EL, Anjos LA. Perfil antropométrico da população brasileira: Resultados da pesquisa nacional sobre saúde e nutrição. 1999.Cadernos de Saúde Pública; 15(4):759-768.

15. Podsiadlo D, Richardson S. 1991. The timed "Up\& Go": a test of basic functional mobility for frail elderly persons. J Am Geriatr Soc.; 39(2):142-8

16. Freitas EV, Miranda RD, Nery MR. Parâmetros Clínicos do EnveIhecimento e Avaliação Geriátrica Global. In: FREITA, E.V. et al. Tratado de Geriatria e Gerontologia. 2002, Rio de Janeiro, RJ: Guanabara Koogan, p. 609-617.

17. Frenk J. Transciones: vidas, instituiciones, ideas. Salud Pública de México. 1997;39(2):144-150.

18. Duncan PW et al., Functional reach: a new clinical measure of balance. Journal of Gerontology. 1990; 45:192-197.

\section{CONCLUSÕES}

As quedas representam graves problemas para os idosos. Nesse estudo pode ser verificada a relação do índice de quedas com o aumento do comprometimento funcional do idoso. Nos resultados foi observada uma associação entre quedas e um fraco desempenho motor, analisado por meio de testes específicos, além de identificar baixos níveis de desempenho motor entre os idosos com quedas recorrentes.

19. Visser $M$ et al. Skeletal muscle mass and muscle strength in relation to lower-extremity performance in older men and women. J Am Geriatr Soc. 2000; 48(4):381-6.

20. Camargos F. Adaptação transcultural e avaliação das propriedades psicométricas da Falls EfficacyScale - International: um instrumento para avaliar medo de cair em idosos [dissertação] Belo Horizonte: Programa de Pós-Graduação em Ciência da Reabilitação da Escola de Educação Física, Fisioterapia e Terapia Ocupacional, Universidade Federal de Minas Gerais; 2007.

21. O'loughlin JL, Robitaille Y, Boivin JF,Suissa S. Incidence of and risk factors for falls and injurious falls among the community-dwelling elderly. American Journal of Epidemiology. 1993; 137(3):342-354

22. Graafans WC, OomsME, Hofstee HM, Bezener PD, Bouter LM Lips $P$. Falls in the elderly: a prospective study of risk factors and risk profiles. Am. J. Epidemiol. 1996; 143(11):1129-1136.

23. Berg WP, Alessio HM, Mills EM, Tong C. Circunstances and consequences of falls in independent community-dwelling older adults. Age and Ageing.1997; 26(4):261-268

24. Perracini MR. Fatores associados a quedas em um coorte de idosos residentes no município de São Paulo. Tese de Doutorado. Escola Paulista de Medicina. Universidade Federal de São Paulo, 2000.

25. Soares, LDA et al. Análise do desempenho motor associado ao estado nutricional de idosos cadastrados no Programa Saúde da Família, no município de Vitória de Santo Antão-PE. Ciênc. Saúde Coletiva, Maio 2012; 17(5):1297-1304.

26. Rogatto GP, Gobbi S. Efeitos da atividade física regular sobre parâmetros antropométricos e funcionais de mulheres jovens e idosas. Revista Brasileira de Cineantropometria e Desempenho Humano. 2001; 3(1):63-69.

27. Guimarães JMN , Farinatti PTV. Análise descritiva de variáveis teoricamente associadas ao risco de quedas em mulheres idosas. Ver Bras Med Esporte. 2005;11(5):299-305.

28. Pijnappels M, Reeves ND. Identification of elderly fallers by muscle strength measures. Eur. J Appl Physiol. 2008;102(5):585-592.

29. Gazzola JM, Perracini MR, Ganança MM, Ganança FF. Fatores associados ao equilíbrio funcional em idosos com disfunção vestibular crônica. Rev Bras Otorrinolaringol.2006; 72(5):683-90.

30. Estefani GAE. Perfil de idosos atendidos em ambulatório de geriatria segundo a ocorrência de queda [dissertação]. Campinas (SP): Universidade Estadual de Campinas; 2007.

31. Cumming RG, Salkeld G, Thomas M, Szonyi G. Prospective study of the impact of fear of falling on activities of daily living, SF-36 scores, and nursing home admission. J Gerontol A Biol Sci Med Sci. 2000; 55(5):299-305

\section{CORRESPONDÊNCIA}

Lisandra Delfino de Albuquerque Soares

Rua Sylla Pessoa de Melo, 18. São Vicente de Paulo, CEP: 55604-125 Vitória de Santo Antão-PE

CEP: 55604-125

E-mail: dlisandra@hotmail.com 